\title{
FONCTIONNEMENT ADVERBIAL ET SENS EN EMPLOI DE À UN POINT (QUE P)
}

\section{Sonia BOUCHAREB}

Université de Sousse, Tunisie

bouchareb.soni@yahoo.fr

Article reçu le 22 mars 2016 | révisé depuis le 12 avril 2016 | accepté le 22 juin 2016

\begin{abstract}
RÉSUMÉ. A un point que est présenté par les dictionnaires de langue, notamment le Trésor de la Langue Française (TLFi) comme une locution adverbiale "assimilable à un adverbe de degré, d'intensité " (article Point). Il s'agit là, en fait, non pas de sa nature mais de l'un de ses emplois. En emploi adverbial, cette séquence en point présente une latitude combinatoire conforme à celle des adverbes de degré. En dépit de ce comportement adverbial, à un point que $P$ est identifié, dans le cadre de cet article, non pas comme une locution adverbiale mais comme une locution prépositionnelle à complément zéro. D'un point de vue sémantique, elle constitue un adverbe de degré tout comme beaucoup, très, extrêmement et énormément toutefois à un point que $P$ véhicule un degré bien plus intense. Ce sens émane, selon nous, de l'association du sens de chacun de ses éléments constitutifs.
\end{abstract}

Mots-clés: adverbe, degré, intensifieur, locution, modifieur, quantifieur, structure interne.

\begin{abstract}
A un point que is defined by language dictionaries, especially by the Trésor de la Langue Française (TLFi), as an adverbial locution « comparable to an adverb of degree or intensity » (Point). In fact this is not a definition of its nature but of one of its uses. In its adverbial usage, this sequence in point presents combinatorial latitude in accordance with adverbs of degree. Despite this adverbial behavior, $\grave{a}$ un point que $P$ is not identified, within the frame of this article, with an adverbial locution but with a prepositional locution with a zero complement. It is an adverb of degree as beaucoup, très, extrêmement and énormément. Yet, à un point que $P$ conveys a more intense degree. According to us, this meaning comes from the combination of the meaning of each constitutive element.
\end{abstract}

Keywords: adverb, degree, intensifier, internal structure, locution, modifier, quantifier.

\section{INTRODUCTION}

À un point que est susceptible de deux emplois : un emploi conjonctif et un emploi adverbial. L'emploi de à un point que comme conjonctif est plus courant en français contemporain que son emploi comme adverbe, avec que relatif objet. Par exemple, selon notre observation sur Frantext, base textuelle sur internet (http://frantext.fr), nous comptons 1695 emplois de à un point que, conjonctif, contre 75 emplois de à un point (que $P$ ) avec que, relatif objet. Nous nous proposons, dans le travail qui suit, de mettre en avant l'emploi adverbial de cette séquence en point, emploi généralement omis par la tradition grammaticale.

L'objectif de cette présente étude est donc de présenter une analyse lexicale, 
syntaxique et sémantique de l'emploi adverbial de à un point (que P).

L'analyse lexicale nous permettra, entre autres, de déduire le degré de figement de cette séquence en point. Nous nous intéresserons aussi, dans notre analyse syntaxique, à la distribution de cette séquence à valeur adverbiale afin de vérifier si celle-ci présente une latitude combinatoire conforme à sa nature. Notre analyse sémantique aura, quant à elle, pour objectif d'étudier la constitution du sens global de l'ensemble de la séquence. Nous verrons que à un point (que $P$ ) a un sens compositionnel tout à fait décelable à travers, notamment, le sens de la préposition à et celui du nom noyau point sans que, pour autant, celui-ci se réduise à la somme des sens individuels de chacun de ses composants.

Le cadre théorique adopté est le distributionnalisme, théorie fondée par Bloomfield (1933) et développée par Harris (1951).

Suivant cette approche distributionnelle, la langue est considérée comme un système " autonome », c'est-à-dire comme un système dont le fonctionnement des formes ne peut être appréhendé que de l'intérieur de la structure qu'elle forme, indépendamment du sens. Pour comprendre le fonctionnement de la langue, il faut partir de l'observation d'un corpus d'énoncés considéré comme un échantillon représentatif de la langue en vue de la description des formes linguistiques qui constituent des éléments accessibles à l'observation directe. Il faut aussi, entre autres, étudier les possibilités combinatoires de ces unités linguistiques afin d'en déduire la distribution, leur(s) position(s) dans la phrase, leur catégorie grammaticale, etc. Les différentes observations d'ordre syntaxique et distributionnel relevées constituent la base linguistique de leur étude sémantique.

Nous avons également eu recours, dans le cadre de notre analyse lexicale de à un point (que P) aux travaux de Gross (1986) et de Gross et Prandi (2004) qui s'inscrivent dans la lignée des travaux de Z. Harris et qui relèvent $\mathrm{du}$ cadre théorique $\mathrm{du}$ lexiquegrammaire. Selon ces deux linguistes, il faut aller au-delà du caractère polylexical et figé des locutions (conjonctives, prépositives, adverbiales, etc.) pour en saisir le fonctionnement réel en langue. Dans notre cas, étudier la constitution interne de à un point que va nous permettre de saisir son degré de figement et l'origine de son sens global.

\section{MÉTHODE}

Dans le cadre de cette présente étude, nous avons opté pour une démarche formelle qui consiste à partir de l'observation des formes linguistiques, issus d'un corpus considéré comme un échantillon représentatif de la langue, pour tenter de comprendre le fonctionnement syntaxique de à un point (que $P$ ) et la constitution de son sens global en emploi.

Ce corpus est composé d'exemples attestés relevant du français contemporain. Ces exemples proviennent majoritairement de textes littéraires issus de Frantext, base textuelle sur internet (http://frantext.fr), parmi lesquels Juliet (1987), Duperey (1999) et de Google livres, moteur de recherche accessible sur internet permettant d'accéder à une bibliothèque numérisée, parmi lesquels Baron (2015), Frot et Jouannelle (2008), Thiery (2014), Boivin (2006), Pellaton (1984), Daudet (1929), Lehman (2006), Dieudonné (1982), Prochasson (2003), Butor et Desoubeaux (1999), Louis-Ferdinand (1974), Baret (2013), Lacordaire (2007), Ferrah (1997), Djaziri (1997), Largeaud (2011), Tremblay (1993), Roland (1915), Péry (2001) et Wagner et Pinchon (1962). Toutefois, nous avons également eu recours à des exemples non littéraires issus de certains sites de Google parmi lesquels "Doctissimo" (s.d.); "La réfraction de la lumière" (s.d.) ; et "Allande" (s.d.), car nous considérons que le corpus attesté ne présente pas obligatoirement toutes les possibilités autorisées par la langue.

Puis, nous avons pris appui sur ces différentes observations pour émettre des hypothèses sur le sens en emploi adverbial de cette séquence en point car, comme le souligne Apresjan (1969) :

De la même manière que les états internes (psychiques) de l'homme se manifestent d'une manière 
physiologique, les «états internes» (les significations) des éléments d'un texte se manifestent dans leur comportement syntaxique, accessible à l'observation directe (p.58).

La méthode adoptée n'est autre que celle consacrée par le distributionnalisme, à savoir la méthode inductive. Le travail du linguiste consiste alors, selon Leeman (1999), "à élaborer, par l'observation des formes et le raisonnement sur les formes observées, une hypothèse sur le sens" (p.75).

\section{RESULTATS ET DISCUSSION}

Comme nous l'avons déjà souligné cidessus, à un point que est, susceptible de deux emplois différents :

1. à un point que a une valeur adverbiale et est présenté par le Trésor de la langue française informatisé (TLFi) comme une locution adverbiale introduite par à, assimilable "à un adverbe de quantité, d'intensité" (article point)

1- J'avais d'ailleurs écrit à son auteur, et il m'avait répondu une lettre qui m'avait touché à un point que je ne saurais dire.

(Juliet, 1987, p.291- 292)

2. à un point que constitue, selon la tradition grammaticale, une locution conjonctive introduisant une consécutive liée à un haut degré d'intensité de la cause exprimée par la proposition principale.

2- Sa tête l'élança un peu à un point qu'il porta sa main à son front.

(Baron, 2015, p.231)

Ces deux emplois sont décelables à partir de la nature de l'élément que: soit que est un pronom relatif (emploi (1)), soit que est une conjonction de subordination (emploi (2)). Dans son emploi adverbial, objet de notre présente étude, à un point que se caractérise par une structure interne particulière, comme nous allons le voir dans le travail qui suit.

\section{Structure interne de à un point que}

Gross (1986, p.11) détermine la structure globale des adverbes (figés ou libres) par la formule générale des groupes nominaux prépositionnels: (A) Prép Dét $N$ Modif où Prép. désigne une préposition introductrice, Dét. un déterminant, $N$ un substantif-noyau et Modif., un modifieur de type adjectival ou phrastique (relative). Gross (1986) précise $\mathrm{qu}^{\prime}$ "il n'est pas nécessaire que les quatre termes de la structure (A) soient simultanément présent" (p.48).

La locution à un point que, et plus précisément à un point que $P$, vérifie cette structure : Prép (à) Dét (un) N (point) Modif. Elle est composée de la préposition $\grave{a}$, de l'article indéfini $u n$, du nom point et d'un modifieur postposé. Il s'agit généralement d'un adjectif (à un point extraordinaire, à un point incroyable, à un point exagéré, etc.) ou d'une structure phrastique de type que $P$ :

3- Découvrir son corps vendredi dernier m'a choqué à un point que vous ne pouvez imaginer.

(Frot et Jouannelle, 2008, p. 396)

Cette phrase peut être dépliée en :

a- Découvrir son corps vendredi dernier m'a choqué à un point.

b-Vous ne pouvez imaginer ce point.

L'élément que est donc un pronom relatif qui assume la fonction COD au sein de la proposition subordonnée relative (que vous ne pouvez imaginer). Nous remarquons que la relative que $P$ est le plus souvent à la forme négative et exprime l'ineffabilité ou l'inconcevabilité du haut degré de l'élément évalué : à un point que je ne saurais dire (ex.1), que vous ne pouvez imaginer (ex. 3), que tu n'imagines pas (ex.4), que souvent nous ne soupçonnons pas (ex.5) qu'on ne peut concevoir (ex.6) :

4- Et maintenant le cancer me torture à un point que tu n'imagines pas!

(Thiery, 2014, p. 15)

5- Mais cette dépendance à notre apparence fait partie de notre vie, pèse sur nous, hommes ou femmes, jeunes ou vieux, beaux ou laids, à un point que souvent nous ne soupçonnons pas. (Duperey, 1999, p.101-102)

6- Au fond, je suis tout le contraire d'un distrait. Et c'est parce que je suis sérieux, à un point qu'on ne peut concevoir, d'un sérieux qui n'autorise aucune frivolité, que je ne fais rien, et que je me refuse à m'employer aussi 
bien dans une profession que dans des loisirs.

(Boivin, 2006, p. 30)

Il s'ensuit donc que la relative que $P$ fait partie intégrante du syntagme nominal (SN) à noyau nominal point : à + SN [( Dét $($ un $)+N($ point $)+$ Modif. $($ Que P) $]$.
Cette séquence en point se présente, comme l'a relevé Gross (1986, p.11), sous la forme d'un syntagme prépositionnel à tête prépositionnel à et s'étend donc à l'ensemble : à un point que $P$.

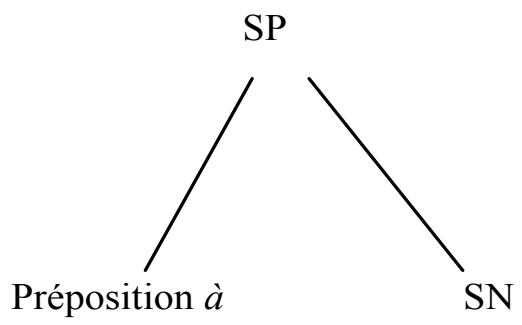

Figure 1. Structure interne de à un point (que P)

L'élément Modif. peut être absent. Cette absence donne lieu à la «locution adverbiale elliptique » (selon la terminologie du TLFi) à un point qui est alors nécessairement suivi du point d'exclamation : à un point !

Selon Gross et Prandi (2004, p.61), l'intonation exclamative est un modifieur qui fait partie intégrante de la détermination du nom noyau point: son absence donne lieu à une phrase incomplète :

7)- a- Ça me dégoûte à un point que tu ne peux imaginer.

(Pellaton, 1984 : 112)

b- *Ça me dégoûte à un point.

Ceci montre que la détermination du nom noyau point est une détermination « composée» (Gavriilidou, 2002, p. 85), formée d'un prédéterminant et d'un modifieur de nature adjectivale ou phrastique (la relative que $P$ ) : UN - Modif. En effet, l'article indéfini $U N$, seul, ne permet pas d'assigner une détermination référentielle complète au nom noyau point.

Le modifieur du nom noyau point relève donc de trois types différents :

1. un adjectif (extraordinaire, incroyable, inimaginable, scandaleux, exagéré, etc.);

2. une relative que $P$ exprimant le haut degré inimaginable $\mathrm{du}$ « point » en question;

3. l'intonation exclamative qui relaie l'absence de que $P$ ou de l'adjectif.

Les éléments susceptibles d'apparaître dans la position Modif. sont certes nombreux mais ils appartiennent à un paradigme sémantique assez restreint: ils expriment tous, d'une manière ou d'une autre, le haut degré indicible du nom point qu'ils caractérisent. Il est à noter d'ailleurs que les autres éléments constitutifs du syntagme prépositionnel (désormais SP) à un point que $P$ sont soit fixes, soit variables dans le cadre d'un paradigme assez réduit comme nous allons le voir dans la partie qui suit.

\section{Étude des possibilités de variations lexicales de la structure à un point que $P$ et degré de figement}

Dans le SP à un point que $P$, la tête prépositionnelle à est fixe: aucune autre préposition n'est envisageable dans la position (Prép.) :

* sur un point que tu ne peux imaginer

* en un point que je ne saurais dire

Il est à remarquer que la séquence en un point que, avec que relatif objet existe en construction libre comme l'atteste l'exemple relevé ci-dessous mais n'existe pas comme structure adverbiale (plus ou moins) figée :

8)- La déviation s'opère juste en un point que l'on appelle point d'incidence.

("La refraction de la lumiere", s.d.)

Lorsque l'article indéfini un est remplacé par l'article défini $l e$, nous passons du syntagme prépositionnel à un point que $P$ à valeur adverbiale à la locution conjonctive $a u$ 
point que, la suite $\grave{a}+l e$ prend alors la forme amalgamée au. Au point que introduit une proposition circonstancielle de conséquence liée au haut degré d'intensité de la cause (être troublé) exprimée par la proposition principale :

9)- a-J'étais troublé à un point que je ne saurais dire.

(Lehman, 2006, p.1146)

b- Je ne l'avais pas publié - car j'étais troublé au point que j'avais besoin de me prouver à moi-même qu'on pouvait faire des mathématiques de façon totalement cohérente.

(Dieudonné, 1982, p.17)

Cette commutation est impossible lorsque le modifieur est un adjectif.

10)- a- Marie triche à un point incroyable. (Gross, 1986, p.413)

b-? Marie triche au point incroyable.

En effet, l'ensemble au point + Adj donne lieu à une phrase inacceptable (exemple $(10$ b)) car elle est incomplète: l'article défini le fait partie intégrante d'une détermination cataphorique de type $L E$ Modif. (Modif. = que $P$, complétive). Elle demeure en suspens même si on ajoute une intonation exclamative $(10 \mathrm{c})$ :

10)- c- ? Marie triche au point incroyable!

Les déterminants $c e$ et tel sont inacceptables dans ce même contexte :

11)- a-Puis après un silence: "Cette arrivée de ma pauvre maman, demain, m'énerve à un point que tu ne saurais croire (...)».

(Daudet, 1929, p. 250)

b-* Cette arrivée de ma pauvre maman, demain, m'énerve à ce/tel point que tu ne saurais croire.

c- * Cette arrivée de ma pauvre maman, demain, m'énerve à ce/tel point incroyable.

d- * Cette arrivée de ma pauvre maman, demain, m'énerve à ce/tel point incroyable!

Le nom point, quant à lui, ne peut commuter qu'avec le nom degré. Cette commutation donne lieu à la séquence à un degré. Selon Gross (1986, p. 402), cet «adverbe » présente la structure (A), avec Modif. = Adj (extraordinairel moindre) et apparaît dans le contexte qui suit: Amuser $N$ hum. à un degré extraordinaire, moindre comme en témoigne l'exemple suivant :

12)- Max amuse Ida, à un degré extraordinaire.

(Gross, 1986, p.174)

Notons, toutefois, que le nom point, noyau nominal de la séquence adverbiale à un point ne peut être modifié que par des adjectifs exprimant le haut degré, voire même un degré extrême alors que le nom degré, noyau nominal de la séquence adverbiale à un degré peut être modifié aussi bien par un adjectif exprimant le haut degré (extraordinaire) que par un adjectif exprimant un degré moindre (moindre, médiocre) :

13)- En effet, l'esprit français, qui ne possède qu'à un degré médiocre ce genre d'imagination, à moins bien su dans le domaine pratique adapter ses œuvre à la réalité, en même temps que dans le royaume du songe il se montrait impuissant.

(Prochasson, 2003, p. 6)

Tout comme le nom-noyau point, le nom-noyau degré peut également être modifié par une relative que $P$ de type : que tu n'imagines pas, que nous ne soupçonnons pas, que tu ne peux concevoir, etc.

14)- Les Américains s'intéressent aux oiseaux à un degré que nous ne soupçonnons pas. Ils jouent un rôle primordial chez eux.

(Butor et Desoubeaux, 1999, p. 184)

Notons aussi, que dans le cadre de cette séquence, le nom point n'est pas pluralisable :

15)-a- Je souffre à un point que je ne saurais dire. (Louis-Ferdinand, 1974, p. 1008)

b- *Je souffre à des points que je ne saurais dire.

La pluralisation du nom point n'est admissible que dans le cadre d'une combinaison libre :

16)- Chaque réunion organisée vous donne droit à des points que vous cumulez sur votre carte de fidélité. ("Allande", s.d.) 
Cette invariabilité en nombre est due, selon Mejri (1997, p.470), à la perte de référentialité du nom.

Il apparaît donc que le SP à un point que $P$ constitue une séquence semi-figée. Le figement, d'une part, de la préposition introductrice à et, d'autre part, l'existence de contraintes pesant sur les autres éléments constitutifs du SP (à un point que P) en font une locution. Il s'agit d'une locution prépositionnelle (désormais LP) à valeur adverbiale composée des éléments constants : $\grave{a}+u n+$ point. Le nom noyau point peut être élargi, comme nous l'avons déjà signalé, par une expansion de type adjectival ou phrastique (une relative). Tout comme la préposition, la LP sous - catégorise un complément qui peut être de différents types. La gamme des compléments d'une préposition se réduit à quatre types différents: un GN, un infinitif, une sousphrase ou l'élément zéro noté (ø). Dans le cas de à un point, cette LP peut être suivie d'un complément phrastique de nature que $P$ (complétive) ou être suivie d'un complément zéro (noté ø) qui est généralement considéré comme un signe d'adverbialisation comme le remarque à juste titre Mélis (2003) :

L'absence de complément est pourtant souvent interprétée comme un signe d'adverbialisation, une telle interprétation ne s'impose pas. Il semble au contraire légitime de considérer qu'il y a toujours un complément, mais que sa réalisation peut se faire sous forme d'un élément vide (p.21).

Dans ce même ordre d'idées, Cadiot (1997) affirme qu'il “est loisible également de voir dans l'adverbe une préposition « intransitive »" (p.18).

Il apparaît donc que la différence d'emploi n'est pas une raison suffisante et fiable pour assigner, comme nous l'avons fait au début de cet article, un statut différent (locution adverbiale (emploi (1) et locution conjonctive (emploi (2)) à une seule et même unité linguistique. Il est donc plus judicieux de parler «d'emploi adverbial» ou de «comportement adverbial» concernant la locution prépositionnelle à un point que $P$ et non de la "recatégoriser » comme locution adverbiale. Quel que soit son emploi, à un point (que P) est une locution prépositionnelle.

Nous nous proposons, dans le travail qui suit, d'étudier la distribution de à un point que $P$ en emploi adverbial afin de voir si celle-ci présente une latitude combinatoire conforme à sa nature adverbiale. Nous en déduirons certaines contraintes d'ordre sémantique qui pèsent sur les éléments susceptibles d'être modifiés par cette locution en point.

\section{Distribution de à un point que $P$ en emploi adverbial}

Comme nous l'avons déjà souligné, à un point que $P$ est présenté par les dictionnaires de langue comme étant assimilable à un adverbe de degré, d'intensité, adverbe défini comme "une espèce particulière $d$ 'adverbes de manière [qui] concerne l'intensité d'une action exprimée par un verbe, d'une qualité ou d'une caractéristique exprimées par un adjectif ou un adverbe" (Grevisse , 1993, p. 943 ). D'ailleurs, la locution à un point (!), présentée comme forme elliptique de à un point que $P$, est glosée par l'expression "à un degré qu'on ne peut imaginer" ("Trésor de la langue française informatisé", s.d.).

Il faut toutefois préciser que tous les adverbes de degré n'ont pas la même capacité distributionnelle. Il n'est qu'à voir beaucoup et très qui présentent une distribution complémentaire : beaucoup ne peut modifier qu'un verbe alors que très peut modifier un adjectif, un adverbe mais pas un verbe :

17)-a- Je l'estime beaucoup.

(Wagner et Pinchon, 1962, p. 406)

b- *Je très l'estime.

18)-a- Il travaille beaucoup mieux.

(Wagner et Pinchon, 1962, p. 406)

b- J'ai couru très loin pour en trouver.

(Wagner et Pinchon, 1962, p. 407)

19)- a- Je l'ai trouvé très fatigué.

(Wagner et Pinchon, 1962, p. 407)

b-*Je l'ai trouvé beaucoup fatigué.

Qu'en est-il de la distribution de à un point (que P) ?

L'observation de notre corpus montre que : 
- à un point que $P$ peut porter sur un verbe :

20)- Je t'aime à un point que tu ne soupçonnes même pas. J'ai besoin de toi et je n'imagine pas que tu puisses me quitter, d'ailleurs, je te le permets pas.

(Djaziri, 1997, p. 231)

Les verbes susceptibles d'être modifiés par à un point que $P$ sont des verbes affectifs (aimer, adorer, souffrir, détester,(se) chagriner, être ému, être touché, manquer, désespérer, décourager, etc.) ou des verbes indiquant un changement de qualité (rajeunir, grandir, changer, vieillir). Ce sont des verbes graduables, c'est-à-dire des verbes qui sont susceptibles de varier en degré.

21)- Je veux dire, ce que j'ai fait est atroce. OK, je le concède ! J'en souffre à un point que vous ne pouvez pas imaginer. Mais ça n'est pas un échec ; ça n'est pas un dérapage ; ça n'est pas un raté. C'est même tout le contraire: c'est une conséquence, c'est l'aboutissement, c'est le succès final.

(Baret, 2013, p. 95)

22)- L'intérêt et la confiance ont grandi à un point que vous ne pouvez imaginer [et] qui m'étonne moi-même. Je ne puis donc...adopter votre incertitude à l'égard de l'avenir.

(Lacordaire, 2007 , p. 264)

- à un point que $P$ peut porter sur un adjectif 23)- Matiya, le grand-père, heureux à un point que personne ne pouvait imaginer, avait laissé dire un moment, puis, subitement agacé, se leva et annonça que son petit-fils porterait le nom de son aïeul Abda.

(Ferrah, 1997, p. 99)

Il s'agit généralement d'adjectifs graduables, c'est à dire d'adjectifs "désignant des propriétés que l'on peut posséder à des degrés divers » (Whittaker, 2002 , p.2). La classe des adjectifs graduables est assez difficile à circonscrire d'une manière unanime et précise, il est plus facile de définir cette classe par ce qu'elle n'est pas, c'est-à-dire de recenser la classe des adjectifs non graduables. Celle-ci regroupe les adjectifs de couleurs, les adjectifs à valeur intrinsèquement superlative (divin, stupéfiant, époustouflant, incroyable, exécrable, abominable...) (Milner, 1978, p.280), les adjectifs relationnels (présidentiel, universitaire, équestre...), les adjectifs exprimant, selon Riegel, Pellat, et Rioul (1994), "une qualité non soumise à variation" (p. 361), (carré, circulaire, rond, équilatéral, parallèle, double, triple, équestre, enceinte...), les adjectifs qui, selon Grevisse (1993, p. 550 b), expriment en eux-mêmes une idée de comparaison (aîné, favori, cadet, préféré, principal, prochain, unique, majeur, mineur...), les adjectifs «déictiques/ anaphoriques», qui, selon Whittaker (2002), “attribuent à l'élément qu'ils qualifient une position temporelle ou spatiale par rapport à un autre élément du contexte" (p. 38), tels que suivant, prochain ou dernier, les adjectifs qui sont étymologiquement des comparatifs (antérieur, extérieur, inférieur, intérieur, postérieur, supérieur), des adjectifs qui sont étymologiquement des superlatifs (infime, intime, minime, ultime, extrême et suprême).

Les adjectifs graduables les plus récurrents dans notre corpus sont des adjectifs affectifs tels sérieux, malheureux, triste, heureux, honteux, etc. Ceci est peut être dû au fait que les émotions étant souvent ressenties comme très intenses, elles nécessiteraient, pour en rendre compte aussi fidèlement que possible, un adverbe de degré susceptible d'exprimer son très haut degré d'intensité.

Certains adverbes peuvent, eux aussi, à l'instar des verbes et des adjectifs relevés cidessus, varier en degré. C'est le cas, selon Riegel, Pellat et Rioul (1994), “des adverbes de temps (tôt, tard, bientôt, longtemps...), de lieu (près, loin), des adverbes de manière en ment (mais aussi de quelques formes simples comme volontiers, bien, mal, vite, etc.) et des adjectifs employés comme adverbes “ (p.383). Ces adverbes peuvent être modifiés par les adverbes de degré (très tôt, assez tard, très longtemps...) et admettent les formes comparative et superlative (plus tard que, le plus tard, plus vite que, le plus vite...)

$\grave{A}$ un point que $P$ peut assez rarement porter sur un autre adverbe :

24)- Un col peut évoluer très très vite à un point $\mathrm{qu}^{\prime}$ on $\mathrm{n}^{\prime}$ imagine pas...ou rester bloqué jusque durant le travail... 
("Doctissimo", s.d.)

À un point (que $P$ ) peut également porter sur un nom, dans quel cas, il est suivi de la préposition de et devient déterminant indéfini (à un point de $+\mathrm{GN}$ ) :

25)-Le but de la relation n'est pas d'avoir quelqu'un d'autre pour te compléter, mais de te rendre compte qui tu es. En se préoccupant de l'autre, en priorité, on en arrive obligatoirement à un point de déception, d'incompréhension car c'est une erreur fatale.

(Largeaud, 2011, p. 46)

Notre corpus montre qu'il s'agit généralement de noms abstraits comme lassitude, déception, incompréhension, discrétion, etc. Notons qu'en tant que déterminant indéfini, à un point de ne peut être suivi, tels beaucoup ou énormément d'un nom au pluriel (beaucoup de femmes, énormément de gens mais * à un point de gens)) ou d'un nom collectif (beaucoup de monde, énormément de monde mais *à un point de monde).

Il apparaît donc, que, en tant qu'adverbe de degré, à un point (que $P$ ) institue des contraintes d'ordre sémantique sur les éléments que cette séquence en point modifie. Ce sont généralement des verbes, adjectifs ou adverbes qui s'accommodent sans peine de variations en degré.

La distribution syntaxique de à un point (que $P$ ) permet aussi de nous rendre compte que, selon la nature de l'élément évalué en terme de degré, cette locution est soit un quantifieur soit un intensifieur. Il est généralement admis que les adverbes de degré portant sur des substantifs (à un point est alors suivi de la préposition de) et sur des verbes comme souffrir, manger, grossir, etc. ou des verbes qui réfèrent à des actions réitérables ou duratives (sortir beaucoup, se voir énormément, attendre beaucoup, travailler beaucoup, etc.). sont des quantifiieurs (ou quantitatifs) alors que ceux qui portent sur des adjectifs et des adverbes sont des intensifieurs (ou intensifs). Tel est le cas de à à un point (que $P$ ) comme nous venons de le voir.

Nous nous proposons dans le travail qui suit de voir si ce sens de haut degré émane un tant soit peu du sens de ses composants ou si ses différentes valeurs (quantifieur, intensifieur) ne sont que des effets de sens induit par la nature des éléments sur lesquels porte la locution à un point (que P).

\section{De la constitution du sens de à un point (que P)}

À un point (que $P$ ) équivaut à un adverbe de degré qui appartient tantôt à la classe des quantifieurs (lorsqu'il modifie un nom ou un verbe) et tantôt à la classe des intensifieurs (notamment lorsqu'il modifie un adjectif ou un adverbe). On peut donc le remplacer par beaucoup ou énormément (ex. 26), dans le premier cas et par très ou extrêmement dans le deuxième cas (ex. 27):

26)-a-Ça m'humilie à un point que tu ne peux imaginer!

(Tremblay, 1993, p.279)

b-Ça m'humilie beaucoup !

c-Ça m’humilie énormément!

27)-a-L'excellent M. Gb me demande quelquefois de tes nouvelles; il est toujours honnête, aimable, intéressant comme tu l'as vu mais malheureux à un point que je n'aurais pas imaginé.

(Roland, 1915, p. 367)

b-L'excellent M. Gb me demande quelquefois de tes nouvelles; il est toujours honnête, aimable, intéressant comme tu l'as vu mais très malheureux.

c-L'excellent M. Gb me demande quelquefois de tes nouvelles; il est toujours honnête, aimable, intéressant comme tu l'as vu mais extrêmement malheureux.

Bien que la glose avec les adverbes beaucoup (énormément) et très (extrêmement) soit tout à fait possible, il nous semble que le degré dénoté par ces différents adverbes n'est pas le même. Enormément et extrêmement expriment un degré supérieur à celui respectivement de beaucoup et de très. Les deux premiers servent, en effet, «de superlatifs", selon le Trésor de la Langue Française informatisé, aux deux seconds. A un point + Modif. (relative ou adjectif ou intonation exclamative) dénote un degré bien plus grand que celui dénoté par les autres 
adverbes employés : cette séquence en point à valeur adverbiale exprime un degré extrême.

À un point + Modif. exprime un degré d'intensité plus intense que les autres adverbes de degré. En effet, l'association des différents sens véhiculés par chacun de ses éléments constitutifs en fait un adverbe de degré à valeur intensive extrême (emphatisée). En effet, le sens de «degré» véhiculé par le nom point est caractérisé comme extrême par le sens de la préposition $\grave{a}$, comme ineffable et inimaginable par le modifieur et comme particulier par l'article indéfini $u n$.

Chaque élément constitutif semble ainsi participer à l'élaboration du sens global de l'ensemble. En effet, la LP à un point Modif. n'est pas sémantiquement figée comme pourrait le laisser penser l'emploi du terme «locution». Il ne s'agit pas d'une séquence opaque (ou non-compositionnelle), puisqu'il est toujours possible de déduire le sens global de cette locution en point à partir de celui de ses constituants (Mejri, 1997). Effectivement, il ne faut pas perdre de vue qu'il existe, des degrés d'opacité et que l'absence d'opacité d'une séquence figée ne remet pas en cause son figement puisque "l'opacité, tout en étant une caractéristique des séquences figées n'en est pas pour autant un élément définitoire" (Mejri, 2005, p.189).

La préposition à suppose un parcours (ou une progression) orienté "vers un point d'aboutissement" (Riegel, Pellat et Rioul, 1994, p.372). Le nom noyau point, réduit au sens de «degré» (et plus précisément, de «degré dans une évolution » ("Trésor de la Langue Française informatisé", s.d.) précise $q^{\prime} u^{\prime} i l$ s'agit d'une progression sur une échelle de valeurs évaluées en termes de "degré » dans l'ordre de la qualité ou de la quantité.

La préposition à précise que l'on a atteint le terme d'un mouvement, et donc que l'on a atteint le point extrême de l'échelle de valeurs. C'est au locuteur qu'incombe le rôle d'assigner la valeur correspondant à ce degré extrême relatif à la quantité d'une qualité (on parle alors d'intensité) ou de la quantité d'une substance (objets et événements) moyennant la détermination du nom point. La détermination du nom point est, comme nous l'avons déjà souligné, de type discontinu, composée de l'article indéfini un et du modifieur: UN-Modif. Elle permet d'attribuer une détermination référentielle complète au nom auquel elle se rapporte (point). L'article indéfini un marque que le nom point n'a pas encore fait l'objet d'un repérage référentiel et que ce nom est encore indéfini mais il précise aussi que l'occurrence du nom point est singulière et que c'est au modifieur qui suit (détermination cataphorique) que revient le rôle de spécifier ce nom noyau. D'ailleurs, Culioli (1999) précise, dans cet ordre d'idées, que un est "un marqueur de discrétisation" (p.131) et que, par conséquent, en employant cet article indéfini, "on construit une occurrence singulière, différenciée par rapport à d'autres occurrences" (Culioli, 1999, p.131). Cette singularité est précisée ultérieurement par le moyen d'une relative ou d'un adjectif qui exprime le caractère inimaginable du point en question ou d'une intonation exclamative qui renvoie à l'indicible du degré extrême.

Dans l'exemple suivant, le locuteur s'attribue une générosité qui n'est pas quelconque:

28)- Je suis juste en étant bon et bon en étant juste. Par-dessus tout, généreux à un point que vous ne pouvez imaginer!

(Péry, 2001, p. 30)

Sa générosité est particulière car elle a atteint un degré ultime à préciser. Le locuteur avoue son incapacité à l'évaluer car quelle que soit la valeur qu'il pourrait lui assigner, elle serait inadéquate et n'épuiserait pas le dicible. L'ineffabilité du degré extrême (au-delà des mots et de l'imaginable) est confirmée par des relatives telles que je ne saurais dire, que je ne saurais exprimer, que tu ne peux imaginer, etc., par des adjectifs tels incroyable, extraordinaire, etc. ou par l'intonation exclamative. L'exclamation souligne que la valeur à assigner est au-delà des mots : la phrase reste en suspens et l'intensité atteint son point le plus haut.

\section{CONCLUSION}

$\mathrm{Au}$ terme de cette présente étude, il apparaît que à un point que est, conformément à sa structure interne, un syntagme 
prépositionnel composé de la préposition à, $\mathrm{du}$ déterminant indéfini $u n$, du nom noyau point et d'un modifieur. Ce modifieur peut être un adjectif ou une sous-phrase, que $P$ (avec que relatif objet) ou une intonation exclamative. Les différents tests de commutation appliqués à ces éléments constitutifs ont montré que cette séquence en point est semi-figée. $\grave{A}$ un point que $P$ est, en fait, une locution prépositionnelle souscatégorisant un complément zéro à valeur adverbiale et non une locution adverbiale. Elle présente, d'ailleurs, une latitude combinatoire conforme à sa nature adverbiale : elle peut porter sur un verbe, un adjectif et, assez rarement sur un autre adverbe. Il existe néanmoins, comme nous l'avons souligné dans le cadre de cet article, des restrictions de sens portant sur les éléments susceptibles d'être modifiés par à un point que $P$.

$\mathrm{D}^{\prime}$ un point de vue sémantique, nous avons remarqué, que, à un point que $P$ est un adverbe de degré véhiculant un degré d'intensité extrême qui émane de l'association du sens de ses différents composants. Cette locution est donc loin de vérifier le critère d'opacité qui est généralement attribué aux séquences figées. A un point que $P$ exprime l'indicible du degré extrême et renvoie à un degré d'intensité bien plus grand que celui dénoté par beaucoup/ très ou énormément/ extrêmement.

\section{REMERCIEMENTS}

Je remercie Mme. Jacqueline BACHA, directrice $\mathrm{du}$ Laboratoire de Recherches Langues, Discours et Cultures (Institut Supérieur des Sciences Humaines de Jendouba, TUNISIE) pour avoir eu l'amabilité de lire et de corriger mon présent travail. Je remercie également toute personne ayant participé de près ou de loin à l'amélioration de cet article.

\section{RÉFÉRENCES}

[Allande]. (s.d.). Repéré à http://www.allande.fr/Lingerie_Alland e_web/FR/devenir_hotesse.awp

Apresjan, J.D. (1969). Syntaxe et sémantique. Langages, (15), 57- 66.

Baret, J. (2013). Frontières invisibles. Paris: Apopsix.
Baron, C. (2015), Martelin. 1 : Le petit prince de la forêt. Paris : Société des écrivains.

Bloomfield, L. (1933). Language. (trad. fr. Payot, 1970). Londres : Allen et Unwin.

Boivin, J.-L. (2006). Un été devant soi : Essai sur le bonheur. Paris : Le Manuscrit.

Butor, M. et Desoubeaux, H. (1999). Entretiens: quarante ans de vie littéraire.1. Nantes : Joseph K.

Cadiot, P. (1997). Les prépositions abstraites en français. Paris: Armand Colin.

Culioli, A. (1999). Pour une linguistique de l'énonciation: Domaine notionnel. Tome 3. Paris : Ophrys.

Daudet, L. (1929). Le cœur brûle: roman contemporain. Paris : Flammarion.

Dieudonné, J. (1982). Penser les mathématiques. Paris : Seuil.

Djaziri, E.-Ch. (1997). Un poisson sur la balançoire. Lille : Gai-Kitsh- Camp.

[Doctissimo]. (s.d.). Repéré à http://forum.doctissimo.fr/grossessebebe/accouchement/efface-bebeengage-sujet_169725_1.htm

Dumur, J. (1983). Pour tout l'or du monde. Lausanne: L'Age d'Homme.

Duperey, A. (1999). Les chats de hasard. Paris : Seuil.

Ferrah, A. (1997). Kahina: roman historique. Alger : Marinoor.

Frot, P. et Jouannelle, Cl. (2008), Blockbuster. Paris : Odile Jacob.

Gavriilidou, Z. (2002). La détermination des noms de sentiment en grec moderne, Langages, 145, 83- 96.

Grevisse, M. (1993), Le bon usage: 13 e édition refondue par A. Goosse. Gembloux: Duculot.

Gross, M. (1986). Grammaire transformationnelle $d u$ français, 3Syntaxe de l'adverbe. Paris: ASSTRIL.

Gross, G. et Prandi, M. (2004). La finalité, Fondements conceptuels et genèse linguistique. Bruxelles: Duculot.

Harris, Z. (1951), Methods in Structural Linguistics. Chicago: University of Chicago Press.

Juliet, Ch. (1987). Accueils : Journal IV. Paris : P.O.L.

Lacordaire, H-D. (2007). Correspondance. 2. 1840-1846. Paris : Cerf. 
Largeaud, J. (2011). Révélations: Apocalypse. Paris : Books on Demand.

[La refraction de la lumiere]. (s.d.). Repéré à http://sc.physiques.free.fr/htmlfiles/co urs/dejenef/intro_reflex.html

Lehman, S. (2006). Chasseurs de chimères : l'âge d'or de la science-fiction française. Paris: Omnibus.

Louis-Ferdinand, C. (1974). Romans. 4. Paris : Gallimard.

Pellaton, J.-P. (1984). Poissons d'or : nouvelles. Lausanne : L'Age d'Homme.

Péry, A. (2001). Signes du temps : Journal 19861992 (6 vol.). Genève : Labor et Fides.

Prochasson, C. (2003). Une crise anglaise de la pensée française? Les Cahiers $d u$ Centre de Recherches Historiques, 31, 211.

Mejri, S. (1997). Le figement lexical, Descriptions linguistiques et structuration sémantique. Tunis: Publications de la Faculté des Lettres de la Manouba.

Mejri, S. (2005). Figement absolu ou relatif : la notion de degré de figement, $\operatorname{Linx} 53$, 183- 196.
Mélis, L. (2003). La préposition en français. Paris: Ophrys.

Milner, J.-Cl. (1978). De la syntaxe à l'interprétation: Quantité, insultes, exclamations. Paris : Le Seuil.

Riegel, M., Pellat, J-C, et Rioul, R., (1994) Grammaire méthodique du français. Paris: PUF.

[Trésor de la langue française informatisé]. (s.d.). Repéré à http://atilf.atilf.fr//tlf.htm

Thiery, J.F. (2014). Wolf. Plombière-les-bains : Ex Aequo.

Tremblay, M. (1993). Le cœur éclaté. Montréal : Leméac.

Roland, M.-J. (1913-1915). Lettres de Madame Roland (2e vol ; édité par C. Perroud). Paris : Imprimerie nationale.

Wagner, R.-L. et Pinchon, J. (1962), Grammaire $d u$ français classique et moderne, Paris : Hachette.

Whittaker, S. (2002). La notion de gradation, Applications aux adjectifs. Berne, Berlin, Bruxelles, Frankfurt/M., New York, Oxford, Wien: Peter Lang. 